\title{
NORMAL SUBGROUPS CONTAINED IN FRATTINI SUBGROUPS ARE FRATTINI SUBGROUPS
}

\author{
R. B. J. T. ALLENBY
}

To Professor B. H. Neumann on his 70th birthday

\begin{abstract}
We prove that if $N$ is a normal subgroup of the finite group $G$ and if $N \subseteq \Phi(G)$, then there exists a finite group $U$ such that $N=\Phi(U)$ exactly. In particular, we see that the generalizations apparent in the conclusions of several recently stated theorems are illusory.
\end{abstract}

1. Introduction. The present note arose out of an attempt to settle the following question of B. H. Neumann [6]:

Let $N$ be a finite group such that $N=\Phi\left(G^{*}\right)$, the Frattini subgroup of $G^{*}$, for some group $G^{*}$. Is $N$ the Frattini subgroup of some finite group?

A simple plan to get a negative answer to this question is as follows. Let $N$ be a normal subgroup of the finite group $G$ such that $N \subseteq \Phi(G)$. Form the generalized free product $X$ of $G$ and $G_{1}$ (where $\left.G \cong{ }^{\phi} G_{1}\right)$ amalgamating $N$ and $N_{1}(=N, \phi)$ according to $\phi$. Then, by [1] (see, for instance, Theorem 4.1) and [7, Theorem 7.3.16, p. 162] $X$ is an infinite group with $\Phi(X)=N$ exactly. We are thus reduced to finding a finite group $G$ possessing a normal subgroup $N$ such that $N \subseteq \Phi(G)$ and yet $N$ is not the Frattini subgroup of any finite group. Now there are some old theorems (see for example [2]) which can be stated in the following form (all groups being finite):

(1) Let $N \triangleleft G$ have the property . . . Then $N$ cannot be equal to $\Phi(G)$.

In particular (1) holds if $N$ is either of the nonabelian groups of order 8. More recent results (see for example [3], [4], [9]) have been stated in apparently more general form as follows:

(2) Let $N \triangleleft G$ have the property . . . . Then $N$ cannot be contained in $\Phi(G)$.

Comparing (1) and (2) the uninformed reader might be led to suppose that there is known to exist a group $N$ which, whilst it cannot coincide exactly with the Frattini subgroup of any finite group, can, nonetheless, be embedded normally in some finite group $G$ so that $N \varsubsetneqq \Phi(G)$. Such an $N$ would, as observed above, then answer Neumann's question negatively.

It is the object of this note to show that the generalization apparent in (2) is illusory by proving

Received by the editors August 28, 1978.

AMS (MOS) subject classifications (1970). Primary 20D25; Secondary 20D15, 20E30.

Key words and phrases. Frattini subgroup, (generalized) free product, free group, wreath product, alternating group. 0002-9939/80/0000-0104/\$02.00 
ThEOREM. Let $N \triangleleft G(G$ finite) be such that $N \subseteq \Phi(G)$. Then there exists a finite group $U$ such that $N=\Phi(U)$.

2. The main lemma. The proof of the Theorem depends heavily on Lemma 2.3 below. To ease the proof of that lemma we separate from it a couple of preliminary lemmas. No attempt is made to state these two lemmas in the sharpest possible form.

LEMMA 2.1. Let $B$ be a finite group and $N$ a normal subgroup of $B$. If $N_{1} \triangleleft B$ is such that $N_{1} \subseteq N$ and $\operatorname{Fit}\left(N / N_{1}\right)=\langle 1\rangle$ then $\left|\operatorname{Fit}\left(B / N_{1}\right)\right| \leqslant|\operatorname{Fit}(B / N)|$. (Here Fit $X$ denotes the Fitting subgroup of $X$.)

Proof. If $\operatorname{Fit}\left(B / N_{1}\right)=T / N_{1}$ then $T / N_{1} \cap N / N_{1}=\langle 1\rangle$ since, by hypothesis, $N / N_{1}$ has no nontrivial normal nilpotent subgroup. Thus, in mapping $B / N_{1}$ naturally onto $B / N$, we see that $T / N_{1}$ is mapped isomorphically to a normal nilpotent subgroup of $B / N$. Q.E.D.

LEMMA 2.2. Let $F$ be a free group on at least $l$ free generators and suppose $N \triangleleft F$ is such that $|F / N|=l$. Then $F$ has a set of free generators $f_{1}, f_{2}, \ldots$ such that, in the natural map $F \rightarrow F / N$, at most $l$ of the generators $f_{i}$ map nontrivially into $F / N$.

The easy proof is left to the reader.

LEMMA 2.3. Let $G$ be a group, $F$ a finitely generated noncyclic free subgroup of finite index. Then there exists $M \triangleleft G$ such that $M \subseteq F,|F: M|<\infty$ and $\operatorname{Fit}(F / M)=\langle 1\rangle$.

Proof. First note that, since $|G: F|<\infty$, there exist $K \triangleleft G$ such that $K \subseteq F$ and $|F: K|<\infty$. Set $T / K=\operatorname{Fit}(F / K)$ and set $l=|T / K|$. Select $t \in \mathbf{Z}$ such that $t>\max \{2 l /(n-1), 4\}$ where $n(\geqslant 2)$ is the rank of $F$. Now $K$ has the alternating group $A_{t}$ as a homomorphic image. As shown in Theorem 6.3 of [1], $K$ has a characteristic subgroup $L$ such that $K / L$ is the direct product of finitely many copies of the simple group $A_{\boldsymbol{t}}$. In particular $L \triangleleft G$.

Now $|F: L|=|F: K||K: L| \geqslant|T: K||K: L| \geqslant l \cdot t ! / 2$. Hence $L$ is free of rank at least $(n-1) l t ! / 2+1$ (by Schreier's Theorem).

Set $S / L=\operatorname{Fit}(F / L)$. By Lemma $2.1 l_{1}=|S / L| \leqslant l$. Further, if $S$ is free of rank $w$, then $L$ is free of rank $(w-1) l_{1}+1$. Thus

$$
\begin{aligned}
(w-1) l+1 & \geqslant(w-1) l_{1}+1 \geqslant(n-1) l t ! / 2+1 \geqslant(n-1) l t / 2+1 \\
& >\frac{(n-1) l \cdot(2 l /(n-1))}{2}+1=l^{2}+1 .
\end{aligned}
$$

Consequently $w>l+1$.

Thus $|S / L|=l_{1} \leqslant l$ whereas $S$ is free on at least $l+2$ free generators. By Lemma $2.2 S$ has a set of free generators $f_{i}$ such that, in the natural map $\phi$ : $S \rightarrow S / L$, at most $l$ of these free generators map to nonidentity elements of $S / L$. Consider the wreath product $W=A_{5} \sim S / L$. Then $W$ is a split extension of the direct product $D$ of $l_{1}$ copies of $A_{5}$ by $S / L$. 
Consider the diagram

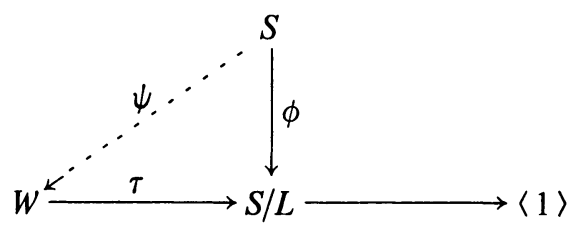

where $\tau: W \rightarrow W / D \cong S / L$. Since $S$ is free of rank $\geqslant l+2$ and since $W$ requires $\leqslant l+2$ generators ( $l$ for $S / L$ and 2 for one of the copies of $A_{5}$ ) we see that the natural map $\phi$ lifts to the epimorphism $\psi$ defined as follows:

(i) If $f_{i} \phi \neq 1$ in $S / L, f_{i} \psi$ is chosen from the preimages of $f_{i} \phi$ under $\tau$.

(ii) From all those $f_{j}$ (there are at least two) such that $f_{j} \phi=1$ select two: say $f_{u}, f_{v}$. Set $f_{u} \psi, f_{v} \psi$ to be the two generating elements of $A_{5}$ mentioned above. Define $f_{j} \psi=1$ in $W$ for any other such $f_{j}$.

Now $W$ has no nontrivial normal nilpotent subgroups. (Clearly no nontrivial normal nilpotent subgroups can meet $D$ nontrivially and it is easy to extend this fact to the whole of $W$.)

Finally we choose $M$ as the intersection of all subgroups $J \triangleleft L$ such that $L / J \cong D$. Then $M$ is characteristic in $L$ and hence $M \triangleleft G$. Further $L / M$ is a direct product of finitely many copies of $A_{5}$.

Now any normal nilpotent subgroup of $F / M$ must lie inside $S / M$ (to prove this just map $F / M$ onto $F / L)$ and hence inside $\operatorname{ker} \psi / M(\operatorname{since} \operatorname{Fit}(S / \operatorname{ker} \psi)=\langle 1\rangle$ ). But this is impossible since $\operatorname{ker} \psi / M \subseteq L / M$ which is a direct product of $A_{5}$ 's and so cannot contain any nontrivial normal nilpotent subgroups. Q.E.D.

3. The theorem. We finally prove the theorem stated earlier.

Let $G$ be any finite group and $N \triangleleft G$ be such that $N \varsubsetneqq \Phi(G)$. Form $X=$ $G *{ }_{1} G_{1}$ as in $\S 1$. Then $X / N=G / N * G_{1} / N$, an ordinary free product. Since $N \varsubsetneqq \Phi(G) \varsubsetneqq G$ we see that each of $G / N$ and $G_{1} / N$ has order at least 4 . By a result of Wielandt [10] we can find a finite simple group, say $Y$, generated by (copies of) $G / N$ and $G_{1} / N$. Then, if $\alpha: G / N * G_{1} / N \stackrel{\text { onto }}{\rightarrow} Y$ is the natural map, we have [5] $\operatorname{ker} \alpha=H$ is a free group, finitely generated because it has finite index in the finitely generated group $X / N$. Further, it is easily seen that $H$ cannot be cyclic. (See, for instance, [8, Satz 8, p. 189]; or look at the centralizer of $H$ in $X / N$.) Thus $X$ is an extension of $N$ by $H$ by $Y$. Now this extension $E$ of $N$ by $H$ splits (since $H$ is free) and so contains a free subgroup, say $F$, isomorphic to $H$ and of finite index in $E$ and hence in $X$. By Lemma 2.3 we can find $M \triangleleft X$ such that $M \subseteq F$, $|F: M|<\infty$ and $\operatorname{Fit}(F / M)=\langle 1\rangle$. Also $X / M$ is an extension of $E / M$ by $Y$ and $E / M$ is itself a split extension of $N$ by $F / M$. Now in the $\operatorname{map} \beta: X \rightarrow X / M$ we know that $\Phi(X) \beta \subseteq \Phi(X \beta)=\Phi(X / M)$. But, by the construction of $F / M$, the simplicity of $Y$ and the fact that the $\Phi$ subgroup of any finite group is a (normal) nilpotent subgroup we see that the $\Phi$ subgroup of $X \beta$ is no larger than $N \beta$. Thus it is exactly $N \beta(\cong N)$ and $X / M$ is the required group $U$. 
4. Comments. (1) As is evident from the above proof, $U$ cannot possibly be a (finite) $p$-group even if $N$ is. This raises once again the question (reiterated in a letter to the author from $\mathrm{H}$. Bechtell):

If $P$ is the Sylow $p$-subgroup of some $\Phi(G)$, is $P$ the Frattini subgroup of some $p$-group?

(2) It would, no doubt, be of interest to finite group theorists to have a proof of the present theorem which does not call upon any infinite group theory. So we raise the question: Can such a 'finite' proof be found? [C. R. B. Wright has found such a proof. See the following paper in these Proceedings.]

(3) We observe that Neumann's question remains unsolved.

\section{REFERENCES}

1. R. B. J. T. Allenby and C. Y. Tang, On the Frattini subgroups of generalized free products, J. Algebra 52 (1978), 460-474.

2. W. Gaschutz, Über die $\Phi$-Untergruppen éndlicher Gruppen, Math. Z. 58 (1953), 160-170.

3. W. Mack Hill and Donald B. Parker, The nilpotence class of the Frattini subgroup, Israel J. Mat. 15 (1973), 211-215.

4. W. Mack Hill and Charles R. B. Wright, Normal subgroups contained in the Frattini subgroup, Proc. Amer. Math. Soc. 35 (1972), 413-415.

5. A. G. Kurosch, Die Untergruppen der freien Produkte von beliebigen Gruppen, Math. Ann. 109 (1934), 647-660.

6. B. H. Neumann, Ascending verbal and Frattini series, Math. Z. 69 (1958), 164-172.

7. W. R. Scott, Group theory, Prentice-Hall, Englewood Cliffs, N. J., 1964.

8. W. Specht, Gruppentheorie, Springer-Verlag, Berlin, 1956.

9. E. L. Stitzinger, A non-embedding theorem for finite groups, Proc. Amer. Math. Soc. 25 (1970), 124-126; Errata, Proc. Amer. Math. Soc. 34 (1972), 631.

10. H. Wielandt, Einbettung Zweier Gruppen in eine einfache Gruppe, Math. Z. 73 (1960), 20-21.

Department of Pure Mathematics, University of Leeds, Leeds, England, United Kingdom 